\title{
The Importance of Tryptophan B28 in H2 Relaxin for RXFP2 Binding and Activation
}

Linda J. Chan ${ }^{1,2}$, John D. Wade ${ }^{* 1,2}$, Frances Separovic ${ }^{2}$, Ross A. D. Bathgate ${ }^{1,3}$ and Mohammed Akhter Hossain ${ }^{* 1,2}$

${ }^{1}$ Florey Neuroscience Institutes, ${ }^{2}$ School of Chemistry, ${ }^{3}$ Department of Biochemistry and Molecular Biology, The University of Melbourne, Victoria 3010, Australia

*Corresponding authors: Mohammed Akhter Hossain and John D. Wade

Florey Neuroscience Institutes, The University of Melbourne, Victoria 3010, Australia; Tel.: +61 (3) 8344 7285; Email: akhter.hossain@florey.edu.au; john.wade@ florey.edu.au; Fax: +61(3) 93481707

Abbreviated title: Tryptophan B28 in relaxin is essential for RXFP2 responses

\section{Abstract}

$\mathrm{H} 2$ relaxin (relaxin) is a member of the insulin-relaxin superfamily and exhibits several nonreproductive functions in addition to its well-known properties as a pregnancy hormone. Over the years, the therapeutic potential of relaxin has been examined for a number of conditions. It is currently in phase III clinical trials for the treatment of acute heart failure. The 53 amino acid peptide hormone consists of two polypeptide chains (A and B) which are cross-linked by two inter-chains and one intra-A chain disulfide bridge. Although its cognate receptor is relaxin family peptide receptor (RXFP) 1 , relaxin is also able to cross-react with RXFP2, for which the native ligand is INSL3. The "RXXXRXXI" motif in the B-chain of H2 relaxin is responsible for primary binding to LRR of the RXFP1 receptor (Büllesbach and Schwabe 2005). Previous RXFP2 receptor mutation and molecular modelling studies strongly suggest that, in addition to this motif, the Trp-B28 residue in the B-chain is responsible for $\mathrm{H} 2$ - 
RXFP2 interaction. To confirm this finding, here we have mutated $\mathrm{H} 2$ relaxin in which TrpB28 was replaced with alanine. The synthetic relaxin analogue was then tested on cells expressing either RXFP1 or 2 to determine the affinity and potency for the respective receptors. Our results confirm that Trp-B28 in the B-chain is crucial for binding and activating RXFP2, but not for RXFP1.

Key words: Relaxin, H2 relaxin, RXFP1, INSL3, RXFP2, Peptide 


\section{Introduction}

The relaxin peptide superfamily in humans is comprised of seven members: relaxin-1 (H1 relaxin), relaxin-2 (H2 relaxin), relaxin-3 (H3 relaxin), insulin-like peptide 3 (INSL3), INSL4, INSL5 and INSL6. In most mammals other than humans and higher primates, there are only two relaxin peptides, relaxin-1 and relaxin-3 and the other insulin-like peptides (INSL3, 4, 5 and 6). In these species, relaxin-1 is the orthologue of $\mathrm{H} 2$ relaxin in humans and higher primates. $\mathrm{H} 2$ relaxin is a pleiotropic homone and is now in phase III clinical trials for the treatment of acute heart failure (Chan et al. 2011).

Like insulin, the heterodimeric peptide hormone relaxin is made up of two chains (A and B chains) and three disulfide bonds (Chan et al. 2011). Its biological effects are manifested through binding to a specific leucine-rich repeat (LRR)-containing G-protein coupled receptor (LGR), LGR7 (Hsu et al. 2002) which has recently been renamed as the

relaxin family peptide (RXFP) receptor 1 (Bathgate et al. 2006). The large extracellular domain of the receptor consists of the leucine-rich repeats (LRR) formed by a series of helices and $\beta$-sheets linked to a low-density lipoprotein receptor type A (LDLa) module (Hsu et al. 2002). RXFP2 is the native receptor for insulin-like peptide 3 (INSL3) (Kumagai et al. 2002), by which cross-reactivity with $\mathrm{H} 2$ relaxin could be possible. Hence, it would be promising to have RXFP1-selective $\mathrm{H} 2$ relaxin analogues in order to reduce undesired side effects mediated through RXFP2 interactions.

The primary binding site of relaxin to RXFP1 involves the interaction between the LRR portion at the ectodomain of the receptor and the binding motif, RXXXRXXI, on the Bchain of the relaxin ligand (Büllesbach et al. 1992; Büllesbach and Schwabe 2000). The primary interaction site in the H2-RXFP1 system has been well-studied and characterized by 
using both mutant ligand (relaxin) and mutant receptor (RXFP1) (Büllesbach et al. 1992; Büllesbach and Schwabe 2000; Büllesbach and Schwabe 2005; Scott et al. 2009).

As mentioned earlier, $\mathrm{H} 2$ relaxin is also able to interact with RXFP2, whose native ligand is INSL3, binding analysis of mutant INSL3 peptides on mutant RXFP2 allowed accurate identification of interaction sites both in the ligand and receptor (Scott et al. 2007). The INSL3 residues involved in RXFP2 binding are His-B12, Arg-B16 and Val-B19 (Rosengren et al. 2006; Scott et al. 2007). The equivalent RXFP2 binding residues in H2 relaxin are Arg-B13, Arg-B17 and Ile-B19 (Scott et al. 2009). These are the same residues by which H2 relaxin also binds to RXFP1 (Büllesbach et al. 1992; Büllesbach and Schwabe 2000). Recent studies on mutant INSL3 and mutant RXFP2 receptor have shown that another residue, Trp-B27, of INSL3 is crucial for RXFP2 binding and activation (Büllesbach and Schwabe 1999; Rosengren et al. 2006; Scott et al. 2007). Similar to the INSL3 ligand, a tryptophan residue is also present on the B-chain of $\mathrm{H} 2$ relaxin. Molecular modelling of the H2-RXFP2 system together with receptor mutation studies suggests that this Trp-B28 in H2 relaxin is involved in RXFP2 receptor binding (Scott et al. 2009). This is suggested by the fact that rhesus monkey relaxin and rat relaxin, which lack a C-terminal tryptophan in their B chains, both exhibit very low affinity for RXFP2 (Scott et al. 2005; Halls et al. 2005a; Halls et al. 2005b). However, there have been no studies carried out using mutant $\mathrm{H} 2$ relaxin to confirm the role of Trp-B28 in receptor binding. Therefore, we have chemically produced a mutant analogue of $\mathrm{H} 2$ relaxin where Trp-B28 was mutated with alanine. The mutant peptide was then tested on RXFP1 and RXFP2 receptor expressing cells. Our results unambiguously confirm that Trp-B28 in H2 relaxin, like Trp-B27 in INSL3, is important for RXFP2 activity and not for RXFP1.

\section{Materials and Methods}




\section{Materials}

9-Fluroenylmethoxtcarbonyl (Fmoc) protected L- $\alpha$-amino acids and 2-(1Hbenzotriazol-1-yl)-1,1,3,3-tetramethyluronium hexafluorophosphate (HBTU) were obtained from GL Biochem (Shanghai). Fmoc-L-Ala-PEG-PS and Fmoc-L-Ser-PEG-PS resins were obtained from Applied Biosystems (Melbourne, Australia). TentaGel S PHB-Cys(Acm) Fmoc was purchased from Rapp Polymere GmbH (Germany). Trifluoroacetic acid (TFA) was obtained from Auspep (West Melbourne, Australia). N,N-dimethylformamide (DMF), dichloromethane (DCM), acetonitrile, piperidine, methanol, diethylether and acetone were purchased from Merck (Melbourne, Australia). 2,2'-dipyridyl disulfide (DPDS) was obtained from Fluka (Switzerland) and trifluoromethanesulfonic acid (TFMSA) from MP Biomedicals (Sydney, Australia). Anisole, 3,6-dioxa-1,8-octanedithiol (DODT), triisopropylsilane (TIPS) and diisopropylethylamine (DIPEA) were obtained from Sigma-Aldrich (Sydney, Australia). AccQTag derivitization kit was purchased from Waters (Sydney, Australia). Dulbecco's modified Eagles' medium (DMEM), RPMI 1640 medium, 2 mM L-glutamine, fetal calf serum and penicillin/streptomycin were all obtained from Trace Biosciences (Sydney, Australia). All other reagents were obtained from Sigma-Aldrich (Sydney, Australia).

\section{Solid-Phase Peptide Synthesis}

Individual $\mathrm{A}$ and $\mathrm{B}$ chains of native relaxin and INSL3, and modified relaxin analogues were synthesized by employing either continuous flow or microwave-assisted solid phase methodologies on an automated PerSeptives Biosystems Pioneer peptide synthesizer and a CEM Liberty peptide synthesizer using appropriate regioselective S-protection strategies. Side chain deprotection and purification of crude A and B chain syntheses were carried out after simultaneous cleavage off the resin supports. The stepwise formation of the three disulfide bridges was conducted through three stages consecutively. Firstly, the oxidation of the A chain, followed by thiolysis and iodolysis subsequently to form the 
respective first and second disulfide bonds between the A and B chains (Hossain et al. 2008; Hossain et al. 2006). All analogues contained two chains (A and B) which are linked covalently by three disulfide bridges.

\section{Peptide Characterization}

Analytical reversed-phase high-performance liquid chromatography was used to assess the purity of individual synthetic peptides on a Vydac C18 column (250x4.6 mm, $300 \AA, 5 \mu \mathrm{m}$ ) with a buffer system of $0.1 \%$ trifluoroacetic acid in water (buffer A) and $0.1 \%$ trifluoroacetic acid in acetonitrile (buffer B). Molecular weights of all analogues were determined by matrix-assisted laser desorption/ionization-time of flight (MALDI-TOF) mass spectrometry on a Bruker Autoflex II instrument (Bremen, Geremany) in the linear mode. Lastly, amino acid analysis utilising vapour-phase acid hydrolysis in $6 \mathrm{M}$ hydrochloric acid containing $2 \%$ phenol at $110^{\circ} \mathrm{C}$ over 24 hours was performed to quantify peptide content. After 24 hours, the peptide hydrolysate was then converted to stable, fluorescent derivatives using the Waters AccQTag kit. The derivitized amino acids were processed through and separated using a Shim-Pak XR ODS column $(3 \times 75 \mathrm{~mm}, 22 \mu \mathrm{m})$ on a Shimadzu microbore RP-HPLC system (Victoria, Australia).

\section{Circular Dichroism}

CD spectra data were recorded using a J-185 JASCO spectrophotometer (Tokyo, Japan) at $25^{\circ} \mathrm{C}$ using a $1 \mathrm{~mm}$ path length cell. The peptides were dissolved in $10 \mathrm{mM}$ phosphate buffer $(\mathrm{pH} 7.5)$ at a concentration of $0.07 \mathrm{mg} / \mathrm{ml}$.

\section{Ligand Binding Assay}

Human embryonic kidney (HEK-293T) cells stably transfected with RXFP1 or RXFP2 were cultured in RPMI 1640 medium (Sigma) supplemented with $10 \%$ fetal calf serum, $100 \mathrm{~g} / \mathrm{mL}$ penicillin, $100 \mathrm{~g} / \mathrm{mL}$ streptomycin and $2 \mathrm{mM} \mathrm{L}$-glutamine and plated into 96-well plates. The 96-well plates were pre-coated with poly-L-lysine for whole cell binding 
assays. Competition binding experiments were carried out using either europium-labeled relaxin (Shabanpoor et al. 2012) or europium-labeled INSL3 in the absence or presence of increasing concentrations of unlabeled relaxin peptide analogues. Fluorescent measurements were recorded at an excitation wavelength of $340 \mathrm{~nm}$ and emission of $614 \mathrm{~nm}$ on a Victor plate reader (Perkin-Elmer). All data were are presented as the mean \pm S.E. of the percentage of the total specific binding of triplicate wells, repeated in at least three separate experiments, and curves were fitted using one-site binding curves in GraphPad Prism 4 (GraphPad Inc, San Diego, CA). Statistical differences in pKi values were analyzed using one-way analysis of variance coupled to Newman Keul's multiple comparison test for multiple group comparisons in GraphPad Prism 4.

\section{Functional cAMP assay}

The evaluation of the ability of native relaxin and INSL3, and other relaxin analogues to stimulate cAMP response was conducted using a cAMP reporter gene assay as described previously (Scott et al. 2006). HEK-293T cells co-transfected with either RXFP1 or RXFP2 and a pCRE $\beta$-galactosidase reporter plasmid were plated in 96-well plates. The cotransfected cells were incubated with increasing concentrations of the relaxin analogue after 24 hours in parallel to $10 \mathrm{nM}$ of relaxin or INSL3 for RXFP1- or RXFP2- transfected cells respectively. The amount of cAMP-driven $\beta$-galactosidase expression in each well was assessed with a colormetric assay measuring absorbance at $570 \mathrm{~nm}$ on a Benchmark Plus microplate spectrophotometer (BioRad). Ligand-induced cAMP stimulation was expressed as a percentage of maximal response of relaxin or INSL3 for RXFP1 and RXFP2 cells respectively. Each data point was measured in triplicate and each experiment conducted independently at least three separate times. Statistical differences in $\mathrm{pEC}_{50}$ values were analyzed using one-way analysis of variance coupled to Newman Keul's multiple comparison test for multiple group comparisons in GraphPad Prism 4. 


\section{Results and Discussion}

There are seven INSL3 primary interaction points in the LRR of RXFP2 (Gln-133, Trp-177, Ile-179, Asp-227, Glu-229, Phe-131 and Asp-181) (Scott et al. 2007; Scott et al. 2009). Interestingly, five of the seven residues (Gln-133, Trp-177, Ile-179, Asp-227, and Glu229) were found to be totally conserved in equivalent positions in RXFP1 and are also part of H2-RXFP1 interactions (Büllesbach and Schwabe 2005). The remaining two residues (Phe131 and Gln-133) in RXFP2 are exclusively part of the INSL3-RXFP2 interactions by which Trp-B27 in INSL3 binds to RXFP2 (Scott et al. 2007). The RXFP2 receptor mutation (mutation at Phe-131 and Gln-133 of RXFP2 residues) and H2-RXFP2 modelling studies suggest that, like Trp-B27 in INSL3, Trp-B28 in H2-relaxin is critical for H2-RXFP2 interaction (Scott et al. 2009). To confirm these findings, we have chemically synthesized a Trp-B28 mutated $\mathrm{H} 2$ relaxin analogue using solid-phase peptide synthesis techniques followed by regioselective disulfide bond formation (Table 1). Two native relaxin family peptides, INSL3 and $\mathrm{H} 2$ relaxin, were also synthesized as controls.

Three different cysteine S-protecting groups (Trt, $\mathrm{tBu}$ and $\mathrm{Acm}$ ) were used to assemble the two-chain peptides to allow regioselective formation of the disulfide bridges. Overall peptide yields of about 15 to $20 \%$ were obtained from the successful synthesis of the peptides. High purities and the correct molecular masses $\left([\mathrm{M}+\mathrm{H}]^{+}\right.$calc: $5846.210 \mathrm{Da}$; $\left.[\mathrm{M}+\mathrm{H}]^{+}{ }_{\text {obs: }} 5847.708 \mathrm{Da}\right)$ of the relaxin peptide analogues were achieved after comprehensive characterization by RP-HPLC and MALDI-TOF mass spectrometry (Figure 1). These analogues were evaluated for their binding capacities and potencies at both RXFP1 and 2 receptors in both receptor -binding and -activation assays. The binding affinities of the relaxin peptides were assessed in the presence of a competitive europium-labelled $\mathrm{H} 2$ relaxin or europium-labelled INSL3 for RXFP1 and RXFP2 receptors respectively. As a means of 
measuring downstream signalling upon ligand-receptor interaction, cAMP responses were measured by absorbance using a colormetric assay.

The $\mathrm{H} 2$ relaxin analogue $\mathrm{H} 2: \mathrm{B}(\mathrm{W} 28 \mathrm{~A})$, which had the Trp-B28 residue replaced by alanine, demonstrated no loss of RXFP1 binding affinity or cAMP activation compared to native $\mathrm{H} 2$ relaxin (Figure 2, Table 2). However, a pronounced loss in RXFP2 binding affinity was observed (Figure 3A, Table 2) which was accompanied by a significant reduction in RXFP2 activation (Figure 3B, Table 2). This result demonstrates the importance of the presence of Trp-B28 in $\mathrm{H} 2$ relaxin for RXFP2 interaction. This finding is supported by our recent study as the truncation of the $\mathrm{C}$-terminal region of relaxin resulted in a significant loss of RXFP2 responses (Hossain et al. 2011). The H2:B(1-24) analogue with the removal of Cterminal region including Trp-B28 displayed significant decrease in binding affinity and activity at RXFP2 (Hossain et al. 2011).

The tryptophan residue at the C-terminus of the INSL3 B-chain was previously replaced by other amino acids such as alanine and leucine, which resulted in reduced affinity, by almost 2 orders of magnitude (Büllesbach and Schwabe 1999). Even mutation of L-Trp to D-Trp showed better binding compared with other amino acids suggesting that the presence of the indole ring of Trp is important. The circular dichroism study confirms that mutation of Trp-B27 to alanine does not alter secondary structure indicating that mutational effects are side-chain driven and not influenced by structure (Büllesbach and Schwabe 1999). Similarly, our CD results displayed subtle differences between the secondary structures of native $\mathrm{H} 2$ relaxin and the analogue (Figure 4). The CD spectra of both peptides revealed a typical $\alpha$ helical conformation with distinct double minima at 208 and $222 \mathrm{~nm}$. Looking at the available X-ray/solution structures (Eigenbrot et al. 1991; Rosengren et al. 2006), the Cterminal helix in $\mathrm{H} 2$ relaxin extends one turn further compared to INSL3. However, the tail following the helix, including the conserved Trp-B28, is more flexible in $\mathrm{H} 2$ relaxin than 
INSL3. Both Trp-B27 in INSL3 and Trp-B28 in H2 relaxin are located outside of the helical region and hence, mutation of this residue in this flexible region should not have any effects in the overall structure of the peptide (Rosengren et al. 2006), as evidenced by CD, suggesting that reduced RXFP2 activity of $\mathrm{H} 2$ relaxin analogue $\mathrm{H} 2: \mathrm{B}(\mathrm{W} 28 \mathrm{~A})$ is side-chain driven and not likely to be influenced by structure.

\section{Concluding Remarks}

We have chemically synthesized an analogue of $\mathrm{H} 2$ relaxin, $\mathrm{H} 2: \mathrm{B}(\mathrm{W} 28 \mathrm{~A})$, where the Trp-B28 residue was replaced with alanine. We showed that the Trp-B28 residue, which is also conserved in the B-chain of INSL3, is crucial for RXFP2 binding and activation. This result confirms our previous observation that relaxin binds to the RXFP2 LRRs utilizing a hybrid relaxin/INSL3 binding site that consists of some of the INSL3-RXFP2 interactions and some of relaxin-RXFP1 interactions in the RXFP2 LRRs (Scott et al. 2009; Scott et al. 2012).

\section{Acknowledgements}

We thank Tania Ferraro and Sharon Layfield for their assistance with the biochemical assays. This work was supported by an NHMRC grant (APP \#1023078) to JDW, RADB and MAH, and (APP \#1023321) to MAH. JDW and RADB are recipients of NHMRC Principle and Senior Research Fellowships, respectively. Studies at the FNI were supported by the Victorian Government's Operational Infrastructure Support Program. 


\section{References}

Bathgate, R. A., Ivell, R., Sanborn, B. M., Sherwood, O. D. and Summers, R. J. (2006) International Union of Pharmacology LVII: Recommendations for the nomenclature of receptors for relaxin family peptides. Pharmacol. Rev, 58, 7-31.

Büllesbach, E. E. and Schwabe, C. (1999) Tryptophan B27 in the relaxin-like factor (RLF) is crucial for RLF receptor-binding. Biochemistry, 38, 3073-3078.

Büllesbach, E. E. and Schwabe, C. (2000) The relaxin receptor-binding site geometry suggests a novel gripping mode of interaction. J. Biol. Chem., 275, 35276-35280.

Büllesbach, E. E. and Schwabe, C. (2005) The trap-like relaxin-binding site of the leucinerich G-protein-coupled receptor 7. J. Biol. Chem., 280, 14051-14056.

Büllesbach, E. E., Yang, S. and Schwabe, C. (1992) The receptor-binding site of human relaxin II. A dual prong-binding mechanism. J Biol Chem, 267, 22957-22960.

Chan, L. J., Hossain, M. A., Samuel, C. S., Separovic, F. and Wade, J. D. (2011) The relaxin peptide family - Structure, function and clinical applications. Protein. Pept. Lett, 18, 220-229.

Eigenbrot, C., Randal, M., Quan, C., et al. (1991) X-ray structure of human relaxin at 1.5 A. Comparison to insulin and implications for receptor binding determinants. J Mol Biol, $221,15-21$.

Halls, M. L., Bathgate, R. A., Sudo, S., et al. (2005a) Identification of binding sites with differing affinity and potency for relaxin analogues on LGR7 and LGR8 receptors. Ann N Y Acad Sci, 1041, 17-21.

Halls, M. L., Bond, C. P., Sudo, S., et al. (2005b) Multiple binding sites revealed by interaction of relaxin family peptides with native and chimeric relaxin family peptide receptors 1 and 2 (LGR7 and LGR8). J Pharmacol Exp Ther, 313, 677-687.

Hossain, M. A., Bathgate, R. A., Kong, C. K., et al. (2008) Synthesis, conformation, and activity of human insulin-like peptide 5 (INSL5). Chembiochem, 9, 1816-1822.

Hossain, M. A., Rosengren, K. J., Samuel, C. S., et al. (2011) The minimal active structure of human relaxin-2. J. Biol. Chem., 286, 37555-37565. 
Hossain, M. A., Zhang, S., Lin, F., et al. (2006) Regioselective disulfide solid phase synthesis, chemical characterization and in vitro receptor binding activity of equine relaxin. Int. J. Pept. Res. Ther., 12, 211-215.

Hsu, S. Y., Nakabayashi, K., Nishi, S., et al. (2002) Activation of orphan receptors by the hormone relaxin. Science, 295, 671-674.

Kumagai, J., Hsu, S. Y., Matsumi, H., et al. (2002) INSL3/Leydig insulin-like peptide activates the LGR8 receptor important in testis descent. J. Biol. Chem., 277, 3128331286.

Rosengren, K. J., Zhang, S., Lin, F., et al. (2006) Solution structure and characterization of the LGR8 receptor binding surface of insulin-like peptide 3. J. Biol. Chem., 281, 28287-28295.

Scott, D. J., Fu, P., Shen, P. J., et al. (2005) Characterization of the rat INSL3 receptor. Ann N Y Acad Sci, 1041, 13-16.

Scott, D. J., Layfield, S., Yan, Y., et al. (2006) Characterization of novel splice variants of LGR7 and LGR8 reveals that receptor signaling is mediated by their unique low density lipoprotein class A modules. J. Biol. Chem., 281, 34942-34954.

Scott, D. J., Rosengren, K. J. and Bathgate, R. A. D. (2012) The different ligand-binding modes of relaxin family peptide receptors RXFP1 and RXFP2. Mol Endocrinol, 26, (In press).

Scott, D. J., Tregear, G. W. and Bathgate, R. A. D. (2009) Modeling the primary hormonebinding site of RXFP1 and RXFP2. Ann. N. Y. Acad. Sci., 1160, 74-77.

Scott, D. J., Wilkinson, T. N., Zhang, S., et al. (2007) Defining the LGR8 residues involved in binding insulin-like peptide 3. Mol. Endocrinol., 21, 1699-1712.

Shabanpoor, F., Bathgate, R. A., Belgi, A., et al. (2012) Site-specific conjugation of a lanthanide chelator and its effects on the chemical synthesis and receptor binding affinity of human relaxin-2 hormone. Biochem. Biophys. Res. Commun, 420, 253256. 


\section{Figure legends}

Figure 1: RP-HPLC profiles and mass spectrometry traces of purified H2:B(W28A) analogue. A) RP-HPLC profile showing peak elution at a retention time of 23.491 minutes. B) Mass spectrometry trace of purified $\mathrm{H} 2: \mathrm{B}(\mathrm{W} 28 \mathrm{~A})$ peptide. The calculated $\mathrm{m} / \mathrm{z}$ value is 5846.210 Da and the actual $\mathrm{m} / \mathrm{z}$ value is $5847.708 \mathrm{Da}$.

Figure 2: Activity of $\mathbf{H} 2$ relaxin analogues with/without alanine mutation on the RXFP1 receptor. A) Competition binding results of native $\mathrm{H} 2$ relaxin, and analogue with alanine mutation, in the presence of a competitive ligand $\mathrm{Eu}^{3+}$-labeled $\mathrm{H} 2$ relaxin tested in HEK293T cells stably expressing RXFP1 receptors. Data are expressed as a percentage of specific binding and are pooled data from at least three experiments performed in triplicate. B) cAMP activity of native $\mathrm{H} 2$ relaxin, and analogue with alanine mutation, in HEK-293T cells expressing RXFP1 receptors using a pCRE-galactosidase reporter gene system. Data are expressed as a percentage of maximum relaxin-stimulated cAMP response and are pooled data from at least three experiments performed in triplicate.

Figure 3: Activity of $\mathbf{H} 2$ relaxin analogues with/without alanine mutation on the RXFP2 receptor. A) Competition binding results of native $\mathrm{H} 2$ relaxin, and analogue with alanine mutation, in the presence of a competitive ligand $\mathrm{Eu}^{3+}$-labeled INSL3 tested in HEK293T cells stably expressing RXFP2 receptors. Data are expressed as a percentage of specific binding and are pooled data from at least three experiments performed in triplicate. B) cAMP activity of native $\mathrm{H} 2$ relaxin, and analogue with alanine mutation, in HEK-293T cells expressing RXFP2 receptors using a pCRE-galactosidase reporter gene system. Data are expressed as a percentage of maximum relaxin-stimulated cAMP response and are pooled data from at least three experiments performed in triplicate.

Figure 4: CD spectra of native $\mathbf{H 2}$ relaxin and $\mathbf{H 2 : B ( W 2 8 A})$. Both peptides were dissolved in $10 \mathrm{mM}$ phosphate buffer at $\mathrm{pH}$ 7.5. 
Table 1: Amino acid sequences of $\mathrm{H} 2$ relaxin, INSL3 and the $\mathrm{H} 2$ relaxin analogue.

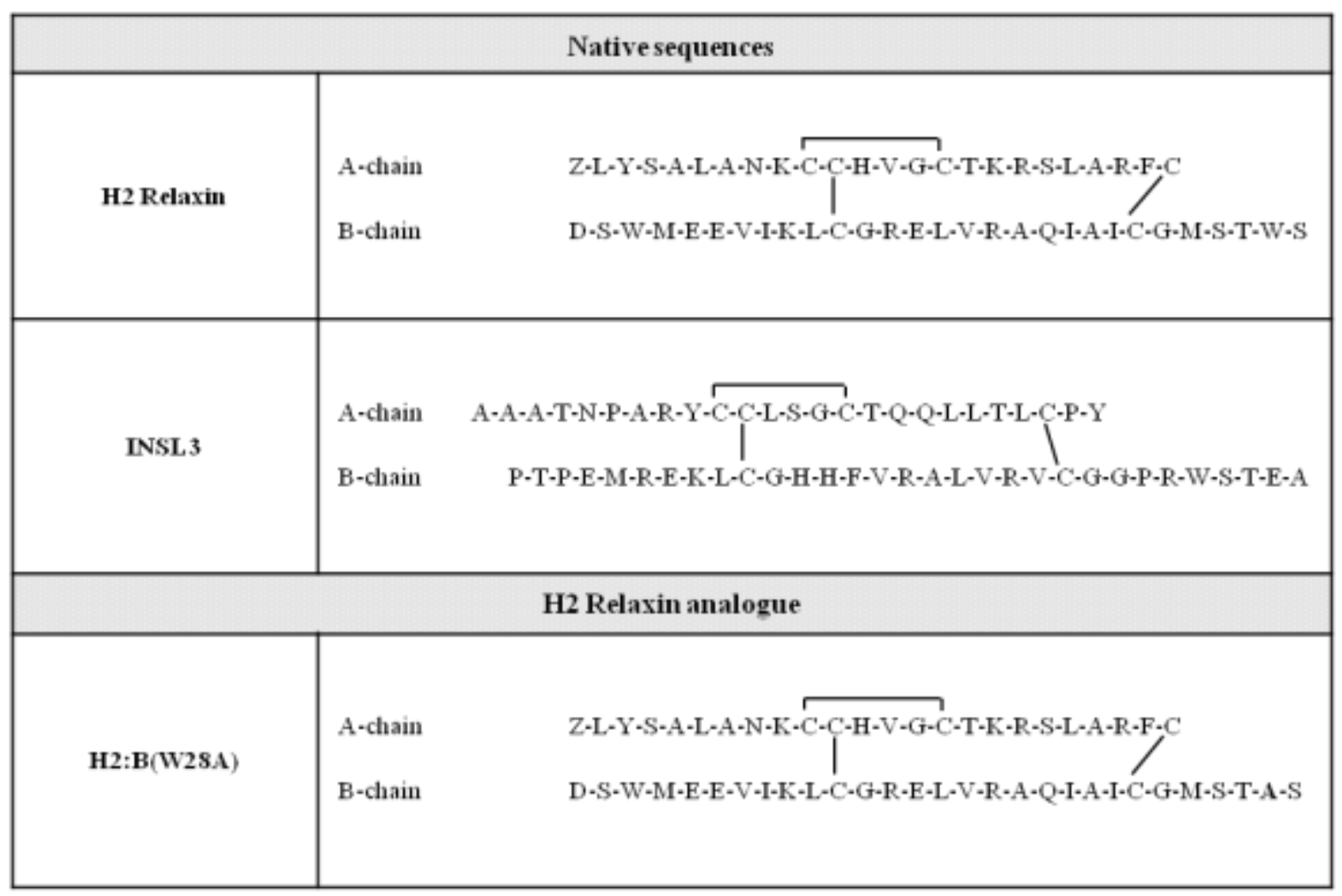


Table 2: Competition Binding $\left(\mathrm{pK}_{\mathrm{i}}\right)$ and Activation $\left(\mathrm{pEC}_{50}\right)$ of RXFP1 and RXFP2 by native $\mathrm{H} 2$ relaxin and its analogue. The values are represented by mean \pm S.E.M.

\begin{tabular}{|c|c|c|c|c|}
\hline & RXFP1 & RXFP2 & RXFP1 & RXFP2 \\
& $\mathbf{p K}_{\mathbf{i}}$ & $\mathbf{p K}_{\mathbf{i}}$ & $\mathbf{p E C}_{\mathbf{5 0}}$ & $\mathbf{p E C}_{\mathbf{5 0}}$ \\
\hline H2 relaxin & $9.74 \pm 0.07$ & $8.59 \pm 0.03$ & $10.71 \pm 0.16$ & $9.14 \pm 0.03$ \\
\hline INSL3 & NA & $9.18 \pm 0.14$ & NA & $10.35 \pm 0.13$ \\
\hline H2:B(W28A) & $9.52 \pm 0.11$ & $6.14 \pm 0.62$ & $10.15 \pm 0.01$ & $6.68 \pm 0.40$ \\
\hline
\end{tabular}


Figure 1:

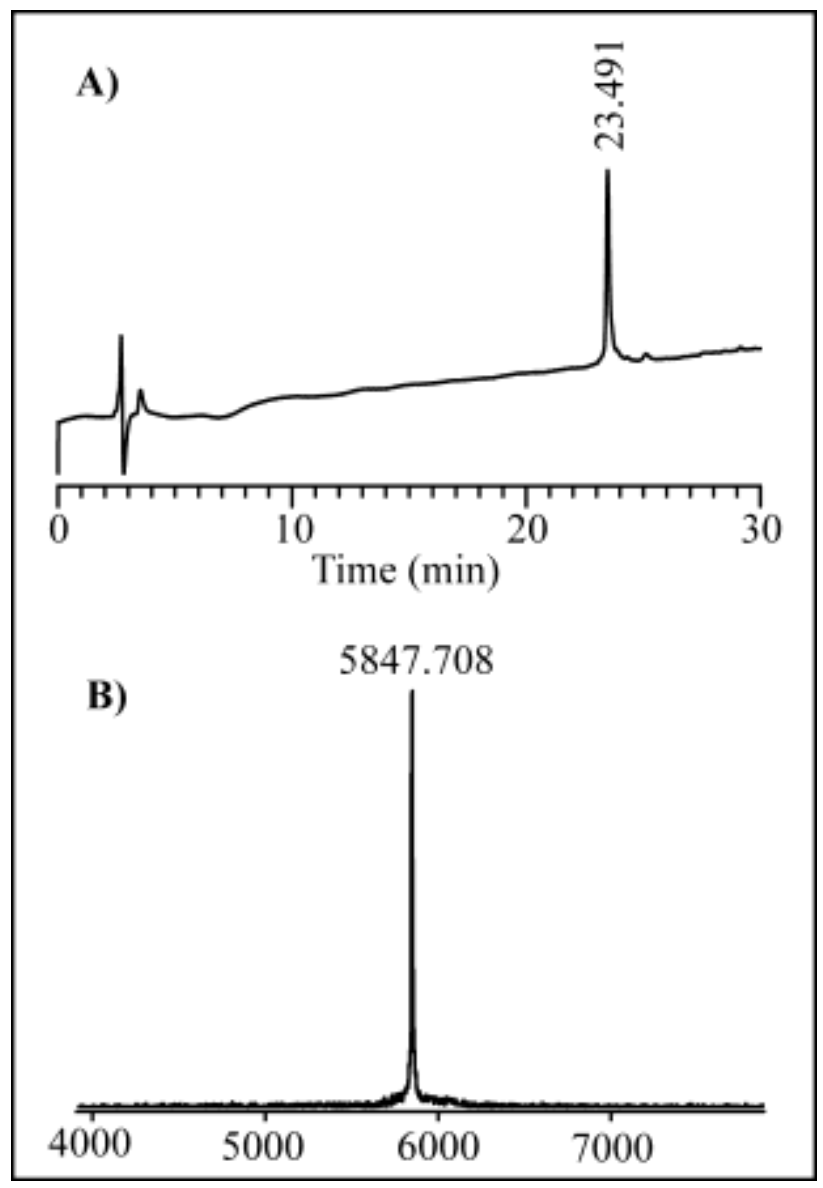


Figure 2:

A)

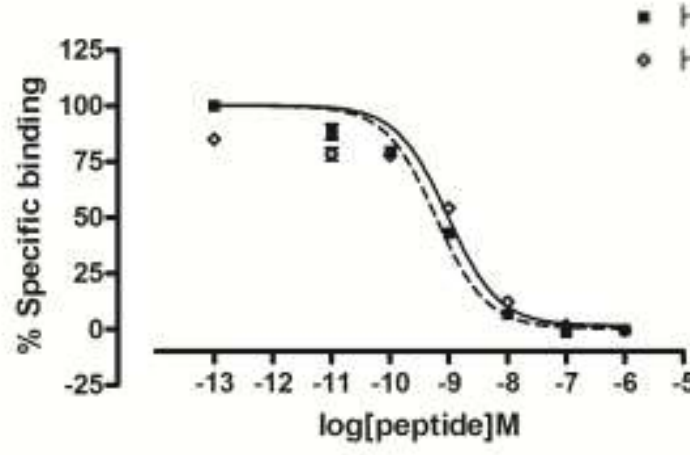

B)

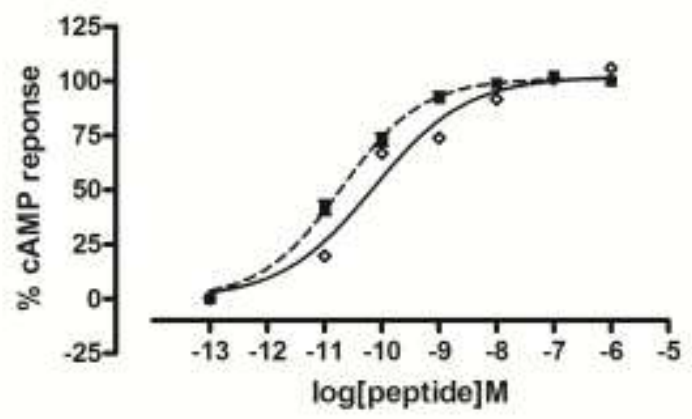

17 
Figure 3:

A)

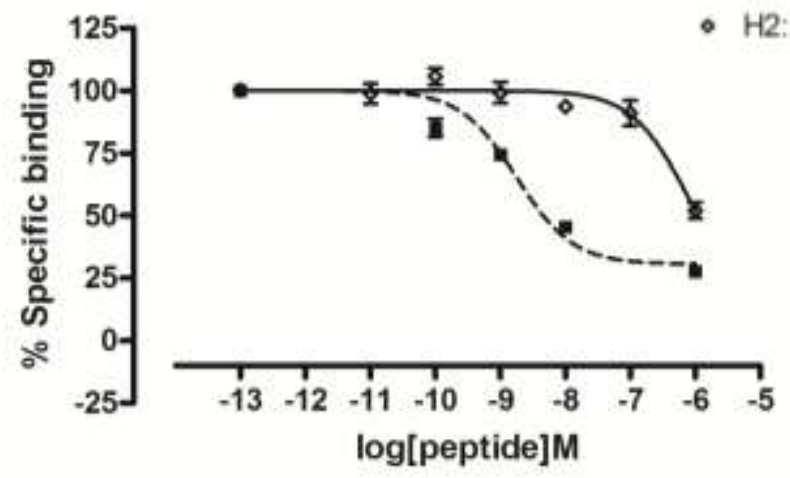

B)

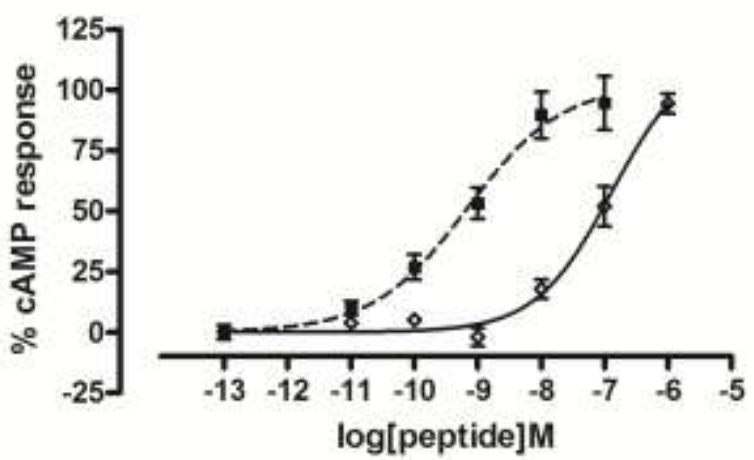


Figure 4:

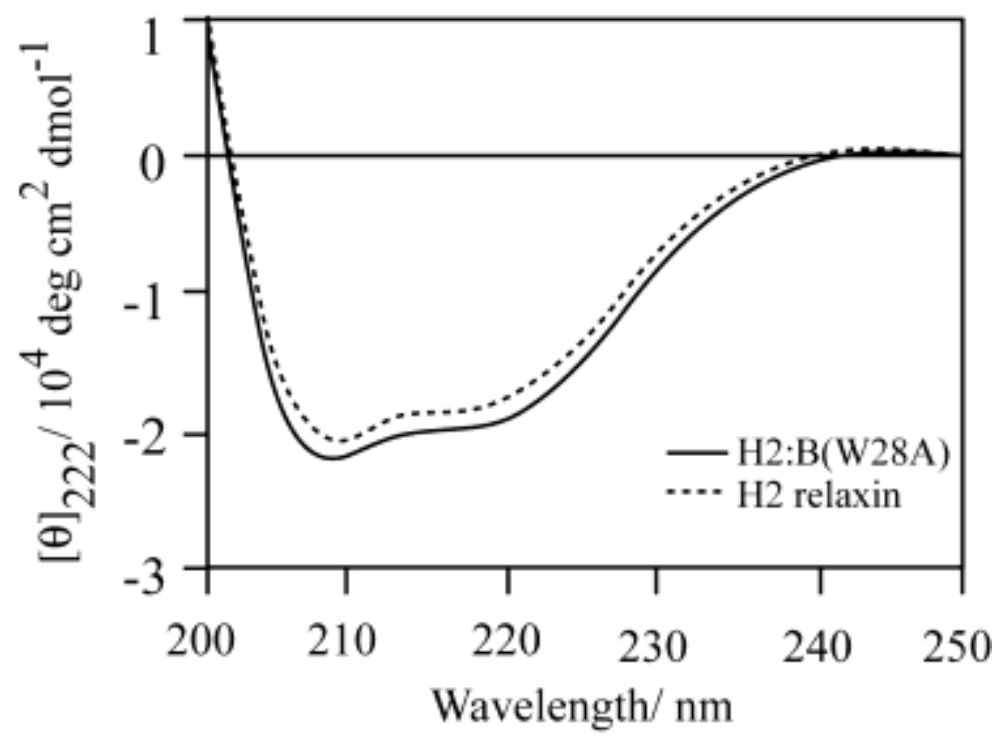

